\title{
SOBRE EL LEGADO DE DAVID STITCHKIN BRANOVER COMO CIVILISTA Y HUMANISTA
}

\author{
ABOUT THE LEGACY OF DAVID STITCHKIN BRANOVER AS A \\ CIVILIST AND HUMANIST
}

JOSÉ LUIS DIEZ SCHWERTER******

\section{RESUMEN}

El presente trabajo expone sobre los hitos relevantes de la vida y obra del profesor de Derecho Civil, David Stitchkin Branover, quien fuera también Rector de la Universidad de Concepción. De los antecedentes biográficos expuestos, se revelan, además de la faceta de destacado jurista, la de un profundo humanismo expresado en las diversas actividades desarrolladas y promovidas durante sus años de docencia (en Concepción y en Santiago), de investigación y de rectorado. Se destaca finalmente su legado jurídico y universitario como de una importancia fundamental en el desarrollo de la intelectualidad penquista y nacional.

Palabras Clave: Jurista chileno; Derecho Civil; Aspectos biográficos; Universidad de Concepción; Rectoría de la Universidad de Concepción; Siglo XX; Humanismo.

\footnotetext{
* Doctor en Derecho. Profesor Titular del Departamento de Derecho Privado, Facultad de Ciencias Jurídicas y Sociales, Universidad de Concepción, Chile. Dirección Postal: calle Víctor Lamas 1290, Barrio Universitario, Casilla 160-C, Concepción, Región del Bio Bio, Chile. Correo electrónico: jdiez@udec.cl.

** Agradezco a los abogados Bernardo Nun y Nicolás Stitchkin la gentileza al proporcionarme valiosa información sobre su familiar David Stitchkin Branover.

*** Una síntesis de este trabajo fue expuesta en el Ciclo Permanente Juristas: La doctrina como fuente del derecho, organizadas por la Facultad de Derecho de la Pontificia Universidad Católica de Chile, específicamente el IX encuentro titulado "Civilistas: Pescio, Caffarena, Stitchkin y Vodanovic", el 2 de diciembre de 2020.
}

Trabajo recibido el 10 de diciembre de 2020 y aceptado para su publicación el 10 de enero de 2021 . 


\section{ABSTRACT}

This work presents the relevant milestones in the life and work of Civil law professor David Stitchkin Branover, who was also Rector of the University of Concepción. Biographical evidence presented, his further facet of prominent jurist reveald a profound humanism, expressed in the various activities developed and promoted during his years of teaching (Concepción and Santiago), research and rector's office. Finally, his legal and university legacy is highlighted as being of fundamental importance in the development of the Concepcion's and Chilean intellectuality.

Keywords: Jurist (legal expert); Civil law; Biographical aspects; University of Concepción; University of Concepcion's Rectorship; XX ${ }^{\text {th }}$ century; Humanism.

\section{PALABRAS PRELIMINARES}

El presente trabajo tiene por objeto destacar aspectos relevantes de la vida y obra del abogado David Stitchkin Branover, profesor de Derecho Civil y quien llegara a ser Rector de la Universidad de Concepción.

$\mathrm{Al}$ respecto, comienzo haciendo un par de prevenciones.

En primer lugar, que el jurista a quien me referiré es originario de Santiago y no de Concepción, ciudad esta última donde pasó menos del veinte por ciento de su vida. Pese a ello, se consideraba un "penquista por adopción" $\mathrm{y}$ en este territorio se le reconoce como una figura gravitante en su historia. ${ }^{2}$

Además, tampoco es sólo un gran civilista, sino además un visionario universitario, un humanista.

La suma de estas características, en un solo personaje, motivan esta exposición, aunque, atendido el contexto y mis conocimientos, ella estará centrada en su faceta de civilista, y refiriéndome sólo a lo más grueso e imprescindible de sus otros aportes e intereses.

\footnotetext{
${ }^{1}$ Academia Chilena de Ciencias Sociales, Políticas y Morales (Eds.), "Entrevista a David Stitchkin”, Revista Societas, 1991, Año I, No 1, pp. 202.

${ }^{2} \mathrm{Al}$ decir de una revista de circulación local: Revista Nos (septiembre 2010). Rostros del Bicentenario: Los 20 guerreros del Biobio. https://www.revistanos.cl/rostros-del-bicentenario-los-20-guerreros-delbiobio/.
} 


\section{SU FORMACIÓN DE ABOGADO EN LA FACULTAD DE DERECHO DE LA UNIVERSIDAD DE CHILE}

David Stitchkin Branover nació en Santiago el 23 de octubre de 1911 siendo hijo único de padres llegados desde Rusia. Su padre falleció tempranamente.

En dicha ciudad completó su educación primaria y secundaria, estableciéndose luego en España junto a su madre y su padrastro (un músico español que vivía en Chile), permaneciendo cuatro años en Sevilla, donde tuvo que desempeñar diversos oficios atendidas las dificultades económicas que encontraron.

Fallecido su padrastro regresan a Chile con su madre, ingresando a estudiar Derecho en la Universidad de Chile en 1932, etapa que asume con especial empeño y dedicación, consciente del sacrificio que ello implicaba a su madre viuda, y con la madurez que le había dado su experiencia europea.

Cabe destacar que en aquel tiempo, y por un programa experimental, los estudios de Derecho tenían una duración de cuatro años, según él recuerda. ${ }^{3}$ Ello en todo caso no fue obstáculo -tal vez no lo sea aún hoy- para que se hayan formado en su generación personajes importantes a quienes recordaba con respeto y aprecio, como Víctor Santa Cruz (con quien tuvo una gran amistad y llegaría a ser profesor de Derecho Civil) y José Miguel Seguel (luego Rector elegido en la Universidad Técnica del Estado). De esta etapa recuerda con afecto a sus profesores de Derecho Civil Enrique Rossel Saavedra y Pedro Lira Urquieta, ${ }^{4}$ al Decano Arturo Alessandri Rodríguez, así como al abogado Gonzalo Barriga Errázuriz, en cuyo estudio trabajó durante su época de estudiante. ${ }^{5}$

David Stitchkin fue el mejor alumno de esa generación, recibiendo los Premios Gormaz y Universidad de Chile. Su sobresaliente Memoria de Prueba sobre "La representación en los actos jurídicos" fue publicada en 1936, con elogiosos informes de Pedro Lira Urquieta y Luis Barriga Errázuriz. ${ }^{6}$ Se titula de abogado en 1937.

Paralelamente a sus estudios, surge su relación con Fanny Litvak

\footnotetext{
${ }^{3}$ Academia Chilena de Ciencias Sociales, Políticas y Morales, cit. (n. 1), p. 193.

${ }^{4}$ Academia Chilena de Ciencias Sociales, Políticas y Morales, cit. (n. 1), p. 193.

${ }^{5}$ Academia Chilena de Ciencias Sociales, Políticas y Morales, cit. (n. 1), pp. 191 y 192.

${ }^{6}$ Stitchkin, David, La representación en los actos jurídicos. Escuela Tipográfica "La Gratitud Nacional", Santiago, 1936.
} 
con quien contraerá matrimonio en 1938, naciendo luego sus cuatro hijos: Sergio, Eliana, Lilian y Claudio.

Por otra parte, ingresará a la Masonería y al Partido Radical.

\section{SU ACTIVIDAD EN LA FACULTAD DE CIENCIAS JURIDICAS Y SOCIALES DE LA UNIVERSIDAD DE CONCEPCIÓN: 1938 A 1946}

Poco tiempo después de titulado de abogado, el azaroso destino lo traerá por primera vez a radicarse al Bio Bío.

Al efecto, una dificultad académica que enfrentaba la Facultad de Ciencias Jurídicas y Sociales de la Universidad de Concepción fue la causa de aquello, y su persona, la elegida para superarla.

En este punto debemos recordar que en 1865 una inquietud presentada en el seno de la Municipalidad de Concepción, respaldada luego por la Intendencia de la zona, motivó la creación por el Estado del "Curso de Leyes del Liceo de Concepción", el cual, tras suprimirse su financiamiento estatal (a fines de 1928), pasó a incorporarse (en 1929), con todos sus cursos, a la naciente Universidad de Concepción, decidida iniciativa ciudadana que bajo la forma de una corporación de derecho privado había sido creada por la Comunidad de Concepción en 1919. Dicha incorporación dio lugar a la Facultad de Ciencias Jurídicas y Sociales de esta Universidad, aunque bajo la tuición académica de la Facultad de Derecho de la Universidad de Chile (como ocurrió hasta 1953 con los establecimientos que impartían tal disciplina en instituciones diversas a la universidad de Bello). ${ }^{7}$

En tal contexto, a mediados de la década del 30' se habían creado la Cátedra de Derecho Civil comparado y profundizado y el Seminario de Derecho Privado. Sin embargo, los resultados obtenidos no fueron los esperados, en especial en un trabajo de investigación que debía efectuarse en cuarto año. Ello motivó al Decano penquista, Alberto Coddou Ortíz, a pedirle a su par de la Universidad de Chile, Arturo Alessandri Rodríguez, que le recomendara un profesor para reforzar esas áreas y superar las dificultades. ${ }^{8}$

\footnotetext{
${ }^{7}$ Sobre la historia de la Facultad de Ciencias Jurídicas y Sociales de la Universidad de Concepción, véase: Pérez, Samuel; RozAs, Sandra, La Facultad de Ciencias Jurídicas y Sociales de la Universidad de Concepción 1865-2015, Universidad de Concepción, Concepción, 2015.

${ }^{8}$ Al respecto: Academia Chilena de Ciencias Sociales, Políticas y Morales, cit. (n. 1), pp. 198 y 199; Pérez y Rozas, cit. (n. 7), pp. 152 y 153.
} 
Fue así como en 1938 llega a Concepción David Stitchkin, haciéndose cargo de la cátedra referida y como Director del Seminario de Derecho Privado.

Las condiciones económicas le permitieron dedicarse tiempo completo a la Universidad (siendo tal vez el primer profesor de Derecho jornada completa en Chile). ${ }^{9}$ Tal desafío implicaba para él - como declaró - una responsabilidad "abrumadora" y a la vez "doble": por una parte "frente a la Escuela de la que egresaba y al Decano que había indicado mi nombre" y por otra "frente a la Universidad que me acogía en su seno". ${ }^{10}$

Serán nueve intensos años en que hará un gran aporte a una Facultad ya heredera, en aquél entonces, de más de setenta años de historia, y en cuyas aulas habían pasado personajes importantes como José Miguel Varela, Juvenal Hernández, Ruperto Bahamonde y Juan Antonio Ríos, por mencionar algunos.

David Stitchkin dará un especial impulso a la investigación jurídica. Y ello desde distintas facetas.

Desde ya como profesor motivó a los estudiantes a sortear con éxito la asignatura mencionada, así como la elaboración de sobresalientes Memorias de Prueba en Derecho Civil, pudiendo mencionarse, entre éstas: Germán Martínez Bustos, Ensayo de una teoría general sobre la renuncia de los derechos: legislación, doctrina y jurisprudencia (1940); Héctor Brain Rioja, La interpretación de los contratos ante la doctrina y jurisprudencia (1941); Bernardo Gesche Müller, El plazo en materia de obligaciones (1941); Héctor Salas Neumann, La estipulación por otro (1941); Hugo Tapia Arqueros, De las obligaciones naturales (1941); Héctor Méndez Eyssautier, Reglas comunes a toda prescripción (1944); Sergio Galaz Ulloa, El contrato de transacción (1944); Luis Egidio Contreras, De la prescripción extintiva civil (1945) y Efraín Vío Vásquez, Las obligaciones condicionales. Doctrina, jurisprudencia y legislación comparada (1945). Mención especial merece la Memoria de Prueba de Orlando Tapia Suárez, De la responsabilidad civil en general y de la responsabilidad delictual entre los contratantes, la cual, publicada originalmente en 1941, mereció ser reimpresa el 2006 por la

\footnotetext{
${ }^{9} \mathrm{Al}$ efecto señaló que "acepté y me fui a Concepción con ¡cinco mil pesos mensuales!, 'full time' y dedicación exclusiva. Gran parte de mi vida, como habrán observado, está en deuda con don Arturo Alessandri”. Academia Chilena de Ciencias Sociales, Políticas y Morales, cit. (n. 1), p. 199.

${ }^{10}$ Stitchkin, David, "Discurso de incorporación de don David Stitchkin Branover como miembro académico de la H. Facultad de Ciencias Jurídicas y Sociales de la Universidad de Concepción", Revista de Derecho Universidad de Concepción, 1961, n 115, año XXIX, p. 200.
} 
Editorial LexisNexis por su vigencia y actualidad, en una iniciativa que tuve el honor de coordinar bajo la atenta revisión de su autor.

El profesor Stitchkin logró estos resultados con dedicación encomiable: sugería temas, encargaba la adquisición de la bibliografía faltante, corregía borradores, y su atención no cesaba hasta la publicación final de estos trabajos. ${ }^{11}$

Pero su legado a la investigación jurídica no sólo se limitó a eso, toda vez que personalmente dio el ejemplo, escribiendo en esta etapa quince artículos en la Revista de Derecho de la Universidad de Concepción. ${ }^{12}$

Destaco desde ya el primero de ellos (publicado en 1938), titulado "Modernas tendencias del derecho", en el cual de manera señera en nuestro medio evidencia el tránsito que se estaba experimentando desde una noción individualista del derecho a una socialización, explicitando diversas proyecciones del fenómeno en instituciones tales como: contratos, imprevisión, abuso del derecho, función social de la propiedad y responsabilidad objetiva, entre otras. ${ }^{13}$

Y, en lo que constituirá una verdadera saga, trece de aquéllos trabajos

${ }^{11} \mathrm{Al}$ respecto puede verse un testimonio en: TAPIA, Orlando, De la responsabilidad civil en general y de la responsabilidad delictual entre los contratantes. Lexis Nexis, Santiago, 2006, $2^{\mathrm{a}} \mathrm{ed}$, pp. XXXIII a XVIV.

${ }^{12} \mathrm{Al}$ respecto: StitchKIn, David, "Notas relativas a la teoría general de las obligaciones", Revista de Derecho Universidad de Concepción, 1939, n² 29-30, año VII, pp. 2351-2416; STITchKIN, David, "Las modernas tendencias del Derecho", Revista de Derecho Universidad de Concepción, 1938, n 23-24, año VI, pp. 1837-1870; STitchKIN, David, "Algunas consideraciones sobre el mandato para ejecutar actos solemnes", Revista de Derecho Universidad de Concepción, 1938, n 25-26, año VI, pp. 20392051; Stitchkin, David, "El mandato civil", Revista de Derecho Universidad de Concepción, 1940, no 33-34, año VIII, pp. 2691-2728; Stitchkin, David, "El mandato civil (continuación)", Revista de Derecho Universidad de Concepción, 1940, nº 35-36, año IX, pp. 2887-2941; STitchKIn, David, "El mandato civil (continuación)", Revista de Derecho Universidad de Concepción, 1941, n 37-38, año IX, pp. 2991-3030; STITCHKIN, David, "El mandato civil (continuación)", Revista de Derecho Universidad de Concepción, 1942, no 39-40, año X, pp. 1-58; STITchкIn, David, "El mandato civil (continuación)", Revista de Derecho Universidad de Concepción, 1944, nº 47, año XII, pp. 1-38; STITchKIn, David, "E1 mandato civil (continuación)", Revista de Derecho Universidad de Concepción, 1944, n 48, año XII, pp. 137-153; Stitchkin, David, "El mandato civil", Revista de Derecho Universidad de Concepción, 1944, no 49, año XII, pp. 249-274; Stitchkin, David, "El mandato civil (continuación)", Revista de Derecho Universidad de Concepción, 1945, no 51, año XIII, pp. 1-27; STITchKIn, David, "El mandato civil (continuación)", Revista de Derecho Universidad de Concepción, 1945, no 52, año XIII, pp. 187-221; STITChKIn, David, "El mandato civil (continuación)", Revista de Derecho Universidad de Concepción, 1945, n 53, año XIII, pp. 225-268; STITchкIN, David, "El mandato civil (continuación)", Revista de Derecho Universidad de Concepción, 1946, n 55, año XIV, pp. 79-121; STITCHKIN, David, "El mandato civil", Revista de Derecho Universidad de Concepción, 1946, n 56, año XIV, pp. 317347.

${ }^{13}$ Stitchin, David, "Las modernas tendencias del Derecho", Revista de Derecho Universidad de Concepción, 1938, nº 23-24, año VI, pp. 1837-1870. 
van a abordar diversos aspectos del mandato civil, investigación que declaró fue preparada en vistas del concurso a Profesor Extraordinario de la Universidad de Chile que rindió mientras era profesor en Concepción. ${ }^{14}$

Por otra parte, en 1938 publicará un artículo en la Revista de Derecho y Jurisprudencia referido a la naturaleza de la representación en los actos jurídicos. ${ }^{15}$

Cabe hacer presente que desde 1941 asumió la dirección de la Revista de Derecho de la Universidad de Concepción, ${ }^{16}$ la cual había sido creada en $1933 .{ }^{17} \mathrm{Y}$, pasado algunos años, pide dispensas para dedicar parte de su tiempo a ejercer de abogado, ${ }^{18}$ en especial asesorando a la Caja de Empleados Públicos y Periodistas.

Pero los intereses de David Stitchkin no se circunscribían sólo a lo jurídico.

En su primera estadía en Concepción se dio tiempo para fundar y ser el primer director de la compañía universitaria de teatro, la cual, con el montaje de "La zapatera prodigiosa" de Federico García Lorca en 1945, marca el origen del Teatro de la Universidad de Concepción (conocido como TUC) el cual en sus 28 años de existencia tendrá gran relevancia en la historia teatral chilena, hasta que fue disuelto en $1973 .{ }^{19}$

A fines de 1946 regresa a Santiago.

${ }^{14}$ Academia Chilena de Ciencias Sociales, Políticas y Morales, cit. (n. 1), p. 207, donde señala que "había rendido examen para optar al título de profesor extraordinario de Derecho Civil de la Universidad de Chile siendo profesor aun de la de Concepción, pues ésta ha sido mi vocación; la educación y la docencia. Con ocasión de ese examen escribí "El Mandato" como tesis para optar al grado de profesor extraordinario".

${ }^{15}$ Stitchkin, David, "Algunas nociones sobre la naturaleza de la representación en los actos jurídicos", Revista de Derecho y Jurisprudencia, T. XXXV, 1ª parte, pp. 113-126.

16 Universidad de Concepción, Memoria del Directorio correspondiente al año 1941. 1942, archivo en formato .pdf, en línea: http://www.archivohistoricoconcepcion.cl/assets/memorias_udec/Memoria UdeC_1941.pdf, pp. 31.

${ }^{17}$ Actualmente es la revista jurídica más antigua en circulación en Chile. La totalidad de sus volúmenes se encuentran digitalizados y consultables en: www.revistadederecho.com.

${ }^{18}$ Academia Chilena de Ciencias Sociales, Políticas y Morales, cit. (n. 1), p. 207.

${ }^{19}$ Sobre la historia del Teatro de la Universidad de Concepción véase: Contreras, Marta; Henríquez, Patricia; Albornoz, Adolfo, Historias del Teatro de la Universidad de Concepción TUC. Trama impresores, Concepción, 2003. 
David Stitchkin se instala en la capital abocándose a una activa labor universitaria y de abogado, en lo que parecía ser una decisión definitiva.

En lo universitario, impartía lecciones en la Universidad de Chile, accediendo a la categoría de Profesor Ordinario de Derecho Civil en 1948. ${ }^{20}$ Ese mismo año publicó el libro "Derecho Civil", en el cual, en dos volúmenes, aborda muy clara y didácticamente el derecho de las obligaciones. ${ }^{21}$

Dos años más tarde publicó su profundo y acabado libro "El Mandato Civil", ${ }^{22}$ el cual constituye hasta hoy un clásico de nuestra literatura jurídica.

También comentará una sentencia en el debatido problema de la tradición del derecho real de herencia. ${ }^{23}$

Paralelamente ejerció activamente la abogacía en la Fiscalía de la Caja Nacional de Empleados Públicos y Periodistas, en diversos organismos públicos, y formando además un estudio jurídico con Gabriel González Videla. En lo gremial integró el Consejo General del Colegio de Abogados de Chile.

Inclusive en su hoja de vida funcionaria en la Universidad de Chile se consigna que en 1948 fue profesor de Interpretación en su Teatro Experimental. ${ }^{24}$

\footnotetext{
${ }^{20}$ Del Río, J. Raimundo, "Memoria del Decano de la Facultad de Ciencias Jurídicas y Sociales de la Universidad de Chile 1946-1954. Separata", Anales de la Facultad de Ciencias Jurídicas y Sociales, 1954, Vol. 1, No 1-3, p. 9. En su actividad docente en esta etapa hay antecedentes de la dirección de la Memoria de Prueba de Luz Bulnes Aldunate (titulada "Interrupción civil de la prescripción adquisitiva" de 1954) como lo recuerda la propia autora en una entrevista: Con KoHAN, Deborah, "Entrevista a Luz Bulnes Aldunate". Revista del Abogado, 2016, n 66, en línea: http://colegioabogados.cl/wp-content/ uploads/2017/05/ABOGADO-66-WEB.pdf, pp. 44. Entre sus alumnos en esta época se cuenta Gonzalo Figueroa Yáñez, respecto del cual David Stitchkin señaló que "fue un alumno muy distinguido en mis distantes años de la cátedra de Derecho Civil" (Stitchkin, David, Prólogo al Libro de: Figueroa Yáñez, Gonzalo, El patrimonio, Editorial Jurídica de Chile, Santiago, 1991, p. 13); Figueroa lo calificó, a su vez, como "el maestro que llegó a ser mi amigo" (FIgueroA, Gonzalo, Memorias de mis últimos 200 años. Editorial Andrés Bello, Santiago, 2009, pp. 282 y 283).

${ }^{21}$ Stitchin, David, Derecho Civil, Editorial Universitaria S.A. Santiago, 1948, Tomo I y II.

${ }^{22}$ Stitchkin, David, El mandato civil, Editorial Jurídica de Chile, Santiago, 1950, en el cual se consigna como afiliación del autor "Profesor titular de Derecho Civil de la Universidad de Chile; ex Director del Seminario de Derecho Privado y Profesor de Derecho Civil comparado y profundizado de la Universidad de Concepción".

${ }^{23}$ Stitchin, David, "Comentario a sentencia de la Corte Suprema de 18 de marzo de 1948", Revista de Derecho y Jurisprudencia, T. XLVI, $2^{\mathrm{a}}$ parte, sección $1^{\circ}$, pp. 94-98, al pie de página.

${ }^{24}$ Universidad de Chile, "Hoja de vida del funcionario David Stitchkin Branover", documento, s/d., copia obtenida por Oficio UT (O) $\mathrm{N}^{\circ} 204 / 2020$ de 16 de junio de 2020, por ley de transparencia. Otorgada por el Sr. Juan Jimeno Ormeño, Jefe de Unidad de Transparencia de la Universidad de Chile
} 
Todo ello hasta que, por una nueva circunstancia imprevista, regresará al Bio Bío a fines de abril de 1956, esta vez para asumir como Rector de la Universidad de Concepción.

\section{SUS DOS PERIODOS DE RECTOR DE LA UNIVERSIDAD DE CONCEPCIÓN: 1956 A 1962 Y $1968^{25}$}

Un doble empate entre los dos candidatos a suceder al Rector fundador de la Universidad de Concepción, don Enrique Molina Garmendia, lo hacen emerger como hombre de consenso para sus respectivos grupos de adherentes. ${ }^{26}$

El buen recuerdo que había dejado en sus nueve años como profesor en la Facultad de Ciencias Jurídicas y Sociales estuvo en la base del apoyo transversal que llevó al Claustro Pleno a elegirlo como Rector de la Universidad de Concepción con una muy amplia mayoría. ${ }^{27}$

El 30 de abril de 1956 llega a Concepción para ejercer por seis años como Rector, en los cuales se manifestará su adelantada visión universitaria y humanista.

Al efecto, impulsará una muy profunda y pionera reforma académica a la Universidad.

Para emprender dicha tarea tuvo en cuenta ciertos factores que, a su entender, la hacían posible: se trataba de una universidad ya asentada y en buena medida financiada (principalmente a través de la Lotería de Concepción), emplazada en un "Campus a escala humana" ("de hombres que caminan a pie", subrayaba), con presencia de un buen número de profesores jornadas completas, y con estudiantes que hacían una intensa

\footnotetext{
al Sr. Samuel Pérez Cofré (quien, a su vez, gentilmente me proporcionó dicho documento).

${ }^{25}$ Respecto del primer periodo de Rectorado, véase el minucioso trabajo de MAzzEI, Leonardo, La Universidad de Concepción en tiempos del rector David Stitchkin Branover. Un proyecto de modernización universitaria (1956-1962), Editorial UdeC, Concepción, 2020.

${ }^{26}$ Los que, por cierto, parecían bastante lejanos (y que han sido caracterizados como laicos y católicos, o conservadores y liberales o beatos y masones o derecha e izquierda, aunque ninguno de los grupos tenía estrictamente esas características). Sobre esta elección, los candidatos y adherentes, y la proposición de Stitchkin como hombre de consenso, véase Academia Chilena de Ciencias Sociales, Políticas y Morales, cit. (n. 1), p. 207; MAZZEI, cit. (n. 25), pp. 37-45; TAPIA, cit. (n. 11), pp. XXXVIII y XV.

${ }^{27}$ Como explica Orlando Tapia Suárez "sus dotes intelectuales como docente y realizador y sus grandes condiciones humanas", y "su amplio espíritu de tolerancia" eran garantía suficiente. TAPIA, cit. (n. 11), pp. XXXVIII y XXXIX.
} 
vida universitaria al provenir muy mayoritariamente de fuera de la ciudad. ${ }^{28}$

Se hace además asesorar para ello por expertos de la UNESCO y consigue el apoyo económico de importantes fundaciones internacionales y de la Organización de las Naciones Unidas. De este modo, potenciará las jornadas completas docentes, la creación de Institutos Centrales en Biología, Física, Química y Matemáticas, restructurará planes y programas de carreras existentes y creará otras, fomentará la investigación, la creación de Departamentos, y todo ello con un fuerte compromiso con la cultura y la comunidad. ${ }^{29}$

Dentro de su legado podemos destacar también la construcción del Foro del Campus Universitario (que se transformará en un punto de encuentro de la ciudad con la Universidad), la creación de la Radio Universidad de Concepción, el fortalecimiento y profesionalización del Teatro de la Universidad de Concepción (TUC), ${ }^{30}$ de la Orquesta Sinfónica y del Coro Universitario, el fortalecimiento de la Casa del Arte y de su Pinacoteca; y del Club de Jazz (al cual compró instrumentos y apoyó la realización de pioneros encuentros internacionales del género). ${ }^{31}$ Mención aparte merecen los talleres y encuentros nacionales e internacionales de escritores y las Escuelas de Temporada, las que durante su gestión lograron convocar, con el motor y visión de Gonzalo Rojas, a un sorprendente número de personalidades en el campo de las humanidades, las artes y las ciencias. ${ }^{32}$

Por otra parte, durante su gestión se contrata a artistas de diversas disciplinas a estancias creativas, y sin que para ello haya sido un obstáculo su eventual falta de formación universitaria. Un notable ejemplo al respecto, es el caso de Violeta Parra (gran artista popular, pero con humanidades inconclusas), a quien la Universidad financió un periodo en Concepción con el propósito de formar un Museo de Música Popular y hacer una recopilación folklórica en la zona. Con esa tranquilidad económica inusual en su vida, la

\footnotetext{
${ }^{28}$ Academia Chilena de Ciencias Sociales, Políticas y Morales, cit. (n. 1), pp. 204, 205, 211 y 212.

${ }^{29}$ Academia Chilena de Ciencias Sociales, Políticas y Morales, cit. (n. 1), pp. 212 y 213.

${ }^{30}$ Que integró, por ejemplo, Pedro de la Barra, Delfina Guzmán, José Chesta, Tennyson Ferrada, Nelson Villagra, Brisolia Herrera, Jaime Vadell, Gustavo Meza, Luis Alarcón, Roberto Navarrete, Lucy Neira, Gustavo Becerra, los hermanos Douvachelle. Sobre la historia del Teatro de la Universidad de Concepción véase: Contreras, Henríquez y Albornoz, cit. (n. 19).

${ }^{31}$ Sobre el desarrollo del jazz en la Universidad de Concepción y el aporte del Rector Stitchkin, véase Universidad de Concepción (Eds.) Académicos en Sintonía IV, Comentarios en Radio Universidad de Concepción, Ed. Universidad de Concepción, Concepción, 2013, pp. 156-158.

${ }_{32}$ Sobre estos encuentros véase el reciente y documentado Bradu, Fabienne, Cambiemos la aldea. Los encuentros de Concepción 1958, 1960, 1962, Fondo de Cultura Económica - Universidad de Concepción, Concepción, 2019.
} 
artista pudo dedicarse a recorrer las entrañas del Biobío, Ñuble e incluso de parte de la Araucanía, dejando registros valiosos para nuestra historia cultural, los que seguramente de otro modo se habrían olvidado por el paso del tiempo. Durante este periodo Violeta Parra realizó además talleres y presentaciones, aunque la idea del museo no se cumplió totalmente. ${ }^{33}$

En lo jurídico, y aun con esta intensa actividad como Rector, David Stitchkin logró publicar algunos ensayos. Así ocurrió con su lección inaugural de las Terceras Escuelas de Verano realizadas en 1957, donde analizó "Los valores permanentes del Derecho". ${ }^{34}$ Por su parte, con ocasión de su incorporación en 1961 como miembro académico de la Facultad de Ciencias Jurídicas y Sociales de la Universidad de Concepción, luego de la intervención del Decano ${ }^{35}$ y de él como homenajeado, ${ }^{36}$ pronunció una conferencia sobre "Los bienes extrapatrimoniales" cuya versión escrita fue publicada en la Revista de Derecho de esta institución, ${ }^{37}$ constituyendo un trabajo señero al reclamar que el concepto de personalidad ampare no sólo intereses pecuniarios o patrimoniales sino también a aquéllos de contenido moral o intelectual, o no patrimonial. ${ }^{38}$ Por su parte ese mismo año Raúl Álvarez Cruz publica en Santiago una versión de sus clases de derecho sucesorio. ${ }^{39}$

Tras finalizar un periodo de rectorado lleno de logros, David Stitchkin

\footnotetext{
${ }^{33}$ Sobre la actividad de Violeta Parra ligada a la Universidad de Concepción, véase el detallado estudio de Venegas, Fernando, Violeta Parra en Concepción y la Frontera del Biobio: 1957-1960. Recopilación, difusión del folklore y desborde creativo. Editorial Universidad de Concepción, Concepción, 2017. En particular sobre la actividad de Violeta Parra en territorio mapuche, véase: MiRANDA, Paula; Loncón, Elisa; Ramay, Allison, Violeta Parra en el Wallmapu. Su encuentro con el canto mapuche, Pehuén, Santiago, 2017.

${ }^{34}$ Dicha lección inaugural de David Stitchkin se reproduce en Monsálvez, Danny, David Stitchkin Branover. Discursos, conferencias, mensajes, entrevistas y clases magistrales. Cuadernos Atenea, Editorial Universidad de Concepción, Concepción, 2014, pp. 284-290 (donde se señala que originalmente estuvo publicada en Revista Atenea 374, pp. 206-216, 1957).

${ }^{35}$ Enríquez, Humberto, "Discurso de recepción del profesor don David Stitchkin Branover como miembro académico de la H. Facultad de Ciencias Jurídicas y Sociales". Revista de Derecho Universidad de Concepción, 1961, nº 115, año XXIX, pp. 189-197.

${ }^{36}$ StitchKin, “Discurso de incorporación...”, cit. (n. 10), pp. 199-205.

${ }^{37}$ Stitchkin, David, "Los Bienes extrapatrimoniales", Revista de Derecho Universidad de Concepción, 1961, nº 115, año XXIX, pp. 3-29.

${ }^{38}$ Este trabajo ha sido destacado recientemente como un aporte relevante en el proceso de "personalización del derecho" experimentada por la dogmática civil chilena; al respecto véase MoraLES, Héctor, "El concepto de persona en el Código Civil: criterios, fundamentos y consecuencias normativas", Ius et Praxis, 2018, Año 24, $\mathrm{N}^{\circ}$ 1, pp. 376-378.

39 Álvarez, Raúl, Derecho Sucesorio (Tomo IV) Según las clases del Profesor Sr. David Stitchkin B. Rector de la Universidad de Concepción, Edugal, Santiago, 1961.
} 
decide no repostular al cargo y regresa a Santiago en 1962, donde retomará el ejercicio profesional (asociado con Gabriel González Videla y Luis Ribalta Puig), así como la docencia universitaria en la Universidad de Chile.

De este periodo destaco especialmente dos trabajos de 1966: uno sobre "El abogado en la Universidad" publicado en la Revista de Derecho y Jurisprudencia, donde reflexiona sobre las funciones de los abogados, la misión de la Universidad y sus interacciones ${ }^{40}$; y otro sobre "Los contenidos esenciales del derecho de propiedad", publicado en la revista "El Campesino" de la Sociedad Nacional de Agricultura, en el cual, comentando el Proyecto de Reforma Constitucional que se tramitaba en aquel entonces (que se traducirá en la publicación de la Ley 16.615 de 20 de enero de 1967), hace un muy claro y agudo análisis de un tema cuya actualidad persiste. ${ }^{41}$

Pero en marzo de 1968 retornará, una vez más, a Concepción, al ser electo nuevamente como Rector por muy amplia mayoría.

Sin embargo, asumirá ahora una Universidad muy distinta, en un contexto ideologizado, de guerra fría y en que, en especial su estudiantado, con la influencia gravitante del MUI (Movimiento Universitario de Izquierda, cercano al MIR, Movimiento de Izquierda Revolucionario) y de otras fuerzas políticas, buscaba avanzar rápidamente a una drástica reforma universitaria en aspectos tales como democracia interna, acceso a la Universidad y rol de ésta en el ámbito político nacional (en sintonía con movimientos semejantes en otros planteles de Chile y el extranjero).

Ante este escenario, David Stitchkin logra aprobar en un breve lapso una reforma profunda a los estatutos universitarios, modificando estructuralmente su orgánica y democracia interna. Concluido ello convoca a elección de Rector, en la que, bajo nuevas reglas, votaban ponderadamente tres estamentos universitarios: académicos, funcionarios no académicos y estudiantes.

Sin embargo, y pese a la insistencia de diversos sectores, declina repostularse y regresa ahora definitivamente a asentarse en Santiago en diciembre de ese año.

¿Por qué no repostuló? En su oportunidad señaló que "circunstancias

\footnotetext{
${ }^{40}$ Stitchkin, David, "El abogado en la Universidad. Conferencia de don David Stitchkin pronunciada el 16 de diciembre de 1966 en el Consejo General del Colegio de Abogados", Revista de Derecho y Jurisprudencia, T. LXIII, $1^{\text {a }}$ parte, pp. 255-265.

${ }^{41}$ Se publicó en Strtchirin, David, "Los contenidos esenciales del derecho de propiedad", El campesino, 1966, Vol. XCVIII, n 3: transcrito en: Garrido, José; Guerrero, Cristián, ValdÉs María Soledad, Historia de la reforma agraria en Chile, Editorial Universitaria, Santiago, 1978, pp. 155-220.
} 
familiares me impiden servir en la Universidad por un nuevo periodo". ${ }^{42}$ En todo caso más de veinte años después dará una más directa y cruda respuesta. ${ }^{43}$ No eran los tiempos para un hombre que creía en la capacidad razonadora y dialogante.

\section{SU REGRESO DEFINITIVO A SANTIAGO DESDE DICIEMBRE DE 1968}

Ya definitivamente en la capital ejercerá activamente la profesión en estudio integrado con su ex alumno y amigo Gonzalo Figueroa Yáñez y su yerno Bernardo Nun Peicihovici.

Como abogado, conocida fue la defensa que realizó de empresas mineras extranjeras ante el Tribunal Especial del Cobre en pleitos en que se debatían deducciones a las indemnizaciones que recibirían del Estado en el proceso de Nacionalización del Cobre, atendidas las rentabilidades excesivas que habrían obtenido mientras operaron en Chile. Eran litigios complejos y como contraparte tenía al Consejo de Defensa del Estado, liderado por su Presidente, el agudo jurista Eduardo Novoa Monreal.

\footnotetext{
${ }^{42}$ En Carta de David Stitchkin dirigida a don Sergio Galaz Ulloa, Secretario General de la Universidad de Concepción de 14 de noviembre de 1968, reproducida en Monsálvez, cit. (n. 34), pp. 188 y 189.

${ }^{43}$ La respuesta está en una entrevista a la Revista Societas. Ante la pregunta ¿cómo se produce la renuncia a la rectoría de la Universidad de Concepción?, responde: "Ahí no fue renuncia, sino que las cosas se dieron de tal forma que pensé... 'En nuestro sistema, en nuestro régimen, somos rectores porque hay un pacto de caballeros. Ni el ejército ni la policía entrarán al campus universitario mientras yo sea rector. Yo no llamaré ejército ni carabineros, pero sí tengo el apoyo del gobierno, no importa'. Entonces, vino la respuesta: 'nosotros respetamos la autonomía universitaria'. 'Eso significa que me voy a sentar a la Plaza de Armas bajo la sombra de unos tilos, a ver lo que ocurre. No, así no. Si es de este modo, vamos a la reforma, la Universidad es autónoma, así será'.

Entonces se produjo la reforma del sistema universitario. Era una reforma política, ya estaban los Van Schouwen, los Enríquez. Edgardo, el padre de Miguel, que era buenísimo y amaba a sus hijos, padecía por ellos, vivía por ellos, vibraba por ellos y naturalmente, si no comulgaba con ellos, por los menos los entendía. Tampoco los apoyaba, pero los entendía. Eran sus hijos, no los iba a dejar abandonados. Les encontraba razones válidas o valederas, para lo que ellos querían. Entonces, después de una visita a La Moneda dije: 'Quieren reforma, vamos a la reforma'. Pero lo hice sabiendo una cosa: cuando hay ciertos sistemas, ciertos métodos para sanar a un enfermo, se tiene que llevar la enfermedad al clímax para ver si el enfermo reacciona... Hubo una película "El pozo de las víboras". Se trata de una mujer que está enferma mentalmente. La historia descansa en el mito de que en la antigüedad, a la gente enferma mentalmente, los tiraban a un pozo de víboras porque el terror los hacía recuperar la razón. Me dije: 'quieren reforma. Este va a ser el pozo de las víboras, van a tener que entender de una vez por todas que eso no puede ser'. Y con el Presidente Frei tramité la reforma. Y dijeron "Bueno, ahora elegimos Rector". Y fueron a verme Miguel Enríquez y Van Schouwen, a pedirme que fuera reelegido Rector y les dije: 'No amigos míos, la comedia e finita, ahora ustedes sabrán', y eligieron a Edgardo Enríquez. Así que no renuncié, terminé anticipadamente mi periodo y no quise ir a la reelección. Esa es la historia, la vida es dramática." Ver en Academia Chilena de Ciencias Sociales, Políticas y Morales, cit. (n. 1), pp. 217 y 218.
} 
Por su parte, en el ámbito universitario ejerce la docencia hasta los 70's en la Universidad de Chile.

De esa etapa interesante resulta ser la versión de sus clases sobre la Parte General del Derecho Civil (simulación, personas y nociones de patrimonio), publicadas en 1969 por Raúl Álvarez Cruz, ${ }^{44}$ y la versión mecanografiada de su Curso de su curso de Derecho Civil profundizado "Teoría general del patrimonio", publicada en 1974, que citarán luego Luis Bustamante Salazar y Gonzalo Figueroa Yáñez al publicar monografías específicas sobre tal argumento, ambas prologadas por el propio Stitchkin. ${ }^{45}$

Sus últimos escritos jurídicos fueron artículos, ${ }^{46}$ de entre los cuales destaco "Ley, derecho y justicia" publicado en 1987, en que de manera clara, madurada y didáctica explica la relación que existe entre esos tres conceptos fundamentales. Como señaló Agustín Squella al reseñarlo: “es hoy un breve texto que conviene leer y meditar. Porque a su rigor teórico e incuestionable proyección sobre problemas jurídicos prácticos de la hora presente, une -además- el propósito testimonial de un jurista y abogado en quien se combinan la erudición y la experiencia". ${ }^{47}$

Por otra parte, la revista Societas de la Academia Chilena de Ciencias Sociales, Políticas y Morales (de la cual fue Miembro de Número y Vicepresidente) le dedicó su sección Conversaciones en 1991, encontrándose ahí material muy valioso para comprender su vida y pensamiento. ${ }^{48}$

En 1980 la Universidad de Concepción le confiere la distinción de Doctor Honoris Causa. ${ }^{49}$

Tras sufrir una larga enfermedad fallece en Santiago, el 12 de Julio de 1997.

\footnotetext{
${ }^{44}$ Álvarez, Raúl, Sintesis de Derecho Civil, (Tomo II) Parte General. Simulación. Las personas y nociones de patrimonio Profesor Sr. David Stitchkin B., Redactados por Raúl Álvarez Cruz, Central de Publicaciones, Centro de Alumnos Escuela de Derecho, Colección de Manuales Jurídicos № 24, Universidad de Chile, 1969.

45 Stitchkin, Prólogo, cit. (n. 20); Stitchkin, David, Prólogo al libro de Bustamante, Luis, El Patrimonio. Dogmática Jurídica, Editorial Jurídica de Chile, Santiago, 1979, pp. 7-11.

${ }^{46}$ Stitchkin, David, Ley, Derecho y Justicia. Cuadernos de Análisis Jurídico no 1 , Escuela de Derecho Universidad Diego Portales, Santiago, 1987; Stitchkin, David, “Adquisición de pagaré", Revista Chilena de Derecho, 1990, Vol. 17, n 1, pp. 211-231, y STiтchкIn, David, "La socialización del Derecho", Anuario de Filosofía jurídica y social, 1990, № 8, pp. 401-403.

${ }^{47}$ SQuella, Agustín, "Reseña a David Stitchkin Branover. Ley, Derecho y Justicia". Anuario de Filosofía Jurídica y Social, 1987, Vol. 5, p. 274.

${ }^{48}$ Academia Chilena de Ciencias Sociales, Políticas y Morales, cit. (n. 1).

${ }^{49}$ El discurso pronunciado por Stitchkin en dicha ocasión se reproduce en Monsálvez, cit. (n. 34), pp. 284-290.
} 


\section{LA PRESENCIA ACTUAL DE DAVID STITCHKIN}

La agudeza y visión del pensamiento jurídico de David Stitchkin lo hacen una figura vigente en nuestro Derecho Civil.

Su libro "El Mandato Civil” acaba de ser reimpreso este 2020 por la Editorial Jurídica de Chile en su quinta edición ${ }^{50}$ actualizada por Gonzalo Figueroa Yáñez (también fallecido), texto que además continúa siendo citado profusamente por la jurisprudencia.

Por su parte, su trabajo "Los contenidos esenciales del derecho de propiedad", fue transcrito en 1979 en el libro "Historia de la reforma agraria en Chile" y su artículo "Las modernas tendencias del Derecho" formó parte del libro "Doctrina civil chilena en el bicentenario 1810-2010", editado por el Departamento de Derecho Privado de la Facultad de Derecho de la Universidad de Chile el 2010. ${ }^{51}$

Por su parte en el marco del Sesquicentenario del Código Civil, su figura fue destacada en una reseña a los profesores de Derecho Civil de la Facultad de Ciencias Jurídicas y Sociales de la Universidad de Concepción, que escribió el Profesor René Ramos Pazos (quien además fue cercano colaborador suyo en la asesoría jurídica de la Universidad en época de Rector y que siempre se encargaba de recordar su figura a las nuevas generaciones)..$^{52}$

Otro tanto ha ido ocurriendo con su preclara visión universitaria.

Así en el año 2000 se le confiere (post mortem) la Distinción Medalla Rectoral en la Universidad de Chile ${ }^{53}$ y en 2012 fue presentado en el Salón Central de la Facultad de Derecho de esa Universidad el libro "Discursos iniciáticos, cátedras magistrales del Rector David Stitchkin", ${ }^{54}$ ocasión en que intervinieron Agustín Squella, Ricardo Lagos y Bernardo Nun. Por

\footnotetext{
${ }^{50}$ Stitchkin, David, El Mandato Civil. Editorial Jurídica de Chile, Santiago, 2020, 5 ad. actualizada por Gonzalo Figueroa Y.

${ }^{51}$ Figueroa, Gonzalo; Tapia, Mauricio; Gatica, María Paz, Doctrina civil chilena en el bicentenario 1810-2010, Universidad de Chile, Santiago, 2010, pp. 159-186.

${ }^{52}$ Ramos, René, "Profesores de derecho civil de la Facultad de Ciencias Jurídicas y Sociales de la Universidad de Concepción durante los últimos 50 años", Revista de Derecho Universidad de Concepción, 2006, № 219-220, año LXXIV, pp. 237-239.

${ }^{53}$ Noticia, "Distinción Medalla Rectoral”, Portal Institucional Universidad de Chile, 2020, disponible en línea: https:/www.uchile.cl/portal/presentacion/simbolos/medallas-y-distinciones/7963/distincionmedalla-rectoral.

${ }^{54}$ Mujica, Francisco; Torres, Federico, Discursos iniciáticos, Cátedras magistrales del Rector David Stitchkin, Salieri Editores, Santiago, 2001.
} 
su parte el año 2014 se editó "David Stitchkin Branover. Compilación de discursos, conferencias, entrevistas y clases magistrales", efectuado por el Profesor Danny Monsálvez en la Universidad de Concepción. ${ }^{55} \mathrm{Y}$ en la última edición de la FILSA (2020) se acaba de presentar un detallado y minucioso libro del Profesor Leonardo Mazzei de Grazia sobre su primer periodo de rectorado. ${ }^{56}$

Otros reconocimientos en el ámbito universitario penquista lo constituyen la inauguración el año 1998 de una escultura confeccionada en su honor por su nieto escultor Javier Stitchkin (denominada "En ascenso"), situada en el Campus Central, así como la instalación de un retrato en su honor en la Facultad de Ciencias Jurídicas y Sociales el año $2001 ;{ }^{57}$ en tanto que el año 2015 la Asociación del Personal Docente y Administrativo de la Universidad de Concepción le asignó el nombre de "Sala David Stitchkin" al remodelado espacio para conferencias, exposiciones y conciertos que dispone en un edificio ubicado al frente de la Plaza de la Independencia en Concepción y que había resultado dañado por el terremoto de $2010 .{ }^{58}$

\section{PALABRAS FINALES}

Conforme se ha expresado en estas líneas, al conocer y ver el resultado del trabajo desarrollado por el Jurista y Rector David Stitchkin, emerge

\footnotetext{
${ }^{55}$ Monsálvez, cit. (n. 34).

${ }^{56}$ MazzeI, cit. (n. 25).

${ }^{57}$ La ceremonia fue encabezada por el Decano de aquél entonces, profesor Sergio Carrasco Delgado, y a ella asistieron, entre otras personalidades, Sergio Stitchkin Litvak, hijo del reconocido Rector y además exalumno de esta Facultad. Sobre este reconocimiento véase: Noticia: "Homenaje a Ex Rector David Stitchkin”, Panorama UdeC. 29 de noviembre de 2001, en línea: http://www2.udec.cl/panorama/ p429/p22.htm. Cabe consignar que el Decano Carrasco además propició en esa época la iniciativa de solicitar a la municipalidad penquista "que dé su nombre a una de las calles de esta ciudad; ojalá cerca de la Universidad, para que sea recuerdo permanente de su personalidad y obra" (CARRASCO, Sergio, "El rector Stitchkin", Columna, El Sur, 10 de diciembre de 2001, p. 2); sin embargo, esta justa iniciativa no ha prosperado a la fecha.

${ }^{58}$ En la ocasión el Presidente de dicha entidad, Profesor Jorge Montecinos Araya, señaló que al elegir el nombre de David Stitchkin para esta sala, se recuerda a un personaje "especialmente significativo para los universitarios, porque fue nuestro segundo Rector, un hombre íntimamente ligado a la Universidad y precisamente a la cultura, al arte, el intelecto, por eso el Directorio propuso la idea (...) también como una forma de hacerle justicia en la memoria de los penquistas que -pensamos- han sido un tanto ingratos con este hombre universitario que tanto le entregó a la ciudad, a la cultura, a las artes". Ver en Noticia: "Reabren Sala Universitaria en el centro de la ciudad", Dirección de Extensión UdeC. 12 de mayo de 2015, disponible en línea: http://extension.udec.cl/reabren-sala-universitaria-en-el-centro-de-la-ciudad/.
} 
la especial virtud que tuvo su vida, cual es, la de ir uniendo mundos, los que, sin su presencia, a veces incluso azarosa y fortuita, podrían haberse desarrollado de forma casi paralela y prácticamente no tocarse.

Desde ya, y circunscribiéndome sólo al área del Derecho, que ha sido el objeto central de este trabajo, fue un jurista destacado que en su prolífica labor formadora estrechó vínculos entre dos escuelas de civilistas: la de la Universidad de Chile y la penquista, ${ }^{59}$ un vínculo que subsiste hasta hoy. Pero su legado fue más allá: acercó las humanidades a las ciencias exactas y a las artes; la capital con el Bio Bío; y nuestra intelectualidad con la del resto de América Latina, aunque sobre estos últimos aspectos, como ya se ha advertido, sólo me haya podido referir a lo más grueso e imprescindible.

\section{BIBLIOGRAFÍA CITADA}

Academia Chilena de Ciencias Sociales, Políticas y Morales (Eds.), "Entrevista a David Stitchkin", Revista Societas, 1991, Año I, No 1, pp. 183-218.

Álvarez, Raúl, Derecho Sucesorio (Tomo IV) Según las clases del Profesor Sr. David Stitchkin B. Rector de la Universidad de Concepción, Edugal, Santiago, 1961.

Álvarez, Raúl, Síntesis de Derecho Civil, (Tomo II) Parte General. Simulación. Las personas y nociones de patrimonio Profesor Sr. David Stitchkin B., Redactados por Raúl Álvarez Cruz, Central de Publicaciones, Centro de Alumnos Escuela de Derecho, Colección de Manuales Jurídicos No 24, Universidad de Chile, Santiago, 1969.

Bradu, Fabienne, Cambiemos la aldea. Los encuentros de Concepción 1958, 1960, 1962, Fondo de Cultura Económica-Universidad de Concepción, Concepción, 2019.

Bustamante, Luis, El Patrimonio. Dogmática Jurídica. Editorial Jurídica de Chile, Santiago, 1979.

CARRASCO, Sergio, "El rector Stitchkin", Columna, El Sur, 10 de diciembre de 2001, p. 2.

Con Kohan, Deborah, "Entrevista a Luz Bulnes Aldunate". Revista del Abogado, 2016, $\mathrm{n}^{\circ}$ 66, en línea: http://colegioabogados.cl/wp-content/

\footnotetext{
${ }^{59}$ Stitchkin califica a la Facultad de Ciencias Jurídicas y Sociales de la Universidad de Concepción como “congénere o hermana de la Universidad de Chile", en Stiтchкin, David, "Discurso de incorporación... “cit. (n. 10), p. 200. Mi colega Mauricio Tapia del Departamento de Derecho Privado de la Universidad de Chile calificó a Stitchkin de "hombre puente" entre estas dos instituciones, en conversación que tuve con él requiriéndole antecedentes sobre la vida académica de David Stitchkin en la Universidad de Chile.
} 
uploads/2017/05/ABOGADO-66-WEB.pdf

Contreras, Marta; Henríquez, Patricia; Albornoz, Adolfo, Historias del Teatro de la Universidad de Concepción TUC. Trama impresores, Concepción, 2003.

Del Río, J. Raimundo, "Memoria del Decano de la Facultad de Ciencias Jurídicas y Sociales de la Universidad de Chile 1946-1954. Separata", Anales de la Facultad de Ciencias Jurídicas y Sociales, 1954, Vol. 1, № 1-3.

ENRíQuez, Humberto, "Discurso de recepción del profesor don David Stitchkin Branover como miembro académico de la H. Facultad de Ciencias Jurídicas y Sociales". Revista de Derecho Universidad de Concepción, 1961, n 115, año XXIX, pp. 189-197.

FigueroA, Gonzalo, El patrimonio. Editorial Jurídica de Chile, Santiago, 1991.

FigueroA, Gonzalo, Memorias de mis últimos 200 años. Editorial Andrés Bello, Santiago, 2009.

FigueroA, Gonzalo; TApia, Mauricio; Gatica, María Paz, Doctrina civil chilena en el bicentenario 1810-2010, Universidad de Chile, Santiago, 2010, pp. 159-186.

MAzzeI, Leonardo, La Universidad de Concepción en tiempos del rector David Stitchkin Branover. Un proyecto de modernización universitaria (19561962), Editorial UdeC, Concepción, 2020.

Miranda, Paula; Loncón, Elisa; Ramay, Allison, Violeta Parra en el Wallmapu. Su encuentro con el canto mapuche, Pehuén, Santiago, 2017.

Monsálvez, Danny, David Stitchkin Branover. Discursos, conferencias, mensajes, entrevistas y clases magistrales. Cuadernos Atenea, Editorial Universidad de Concepción, Concepción, 2014.

Morales, Héctor, "El concepto de persona en el Código Civil: criterios, fundamentos y consecuencias normativas", Ius et Praxis, 2018, Año 24, N 1, pp. 361-396.

Mujica, Francisco; Torres, Federico, Discursos iniciáticos, Cátedras magistrales del Rector David Stitchkin, Salieri Editores, Santiago, 2001.

Noticia: "Reabren Sala Universitaria en el centro de la ciudad", Dirección de Extensión UdeC. 12 de mayo de 2015, en línea: http://extension.udec.cl/reabrensala-universitaria-en-el-centro-de-la-ciudad/.

Noticia: "Homenaje a Ex Rector David Stitchkin", Panorama UdeC. 29 de noviembre de 2001, en línea: http://www2.udec.cl/panorama/p429/p22.htm.

NoтісіA, "Distinción Medalla Rectoral”, Universidad de Chile, 2020, en línea: https://www.uchile.cl/portal/presentacion/simbolos/medallas-y-distinciones/7963/ distincion-medalla-rectoral.

PÉrez, Samuel; Rozas, Sandra, La Facultad de Ciencias Jurídicas y Sociales de la Universidad de Concepción 1865-2015, Universidad de Concepción, Concepción, 2015.

Ramos, René, "Profesores de derecho civil de la Facultad de Ciencias Jurídicas y Sociales de la Universidad de Concepción durante los últimos 50 años", 
Revista de Derecho Universidad de Concepción, 2006, № 219-220, año LXXIV, pp. 237-251.

SQuella, Agustín, "Reseña a David Stitchkin Branover. Ley, Derecho y Justicia". Anuario de Filosofia Jurídica y Social, 1987, Vol. 5, pp. 271-274.

Stitchkin, David, La representación en los actos jurídicos. Escuela Tipográfica "La Gratitud Nacional", Santiago, 1936.

StitchKin, David, "Las modernas tendencias del Derecho", Revista de Derecho Universidad de Concepción, 1938, n 23-24, año VI, pp. 1837-1870.

STITCHKIN, David, "Algunas consideraciones sobre el mandato para ejecutar actos solemnes", Revista de Derecho Universidad de Concepción, 1938, n 25-26, año VI, pp. 2039-2051.

STITCHKIn, David, "Algunas nociones sobre la naturaleza de la representación en los actos jurídicos", Revista de Derecho y Jurisprudencia, T. XXXV, $1^{a}$ parte, pp. 113-126.

STitchKIn, David, "Notas relativas a la teoría general de las obligaciones", Revista de Derecho Universidad de Concepción, 1939, $\mathrm{n}^{\circ}$ 29-30, año VII, pp. 2351-2416.

STITChKIn, David, "El mandato civil", Revista de Derecho Universidad de Concepción, 1940, n 33-34, año VIII, pp. 2691-2728.

Stitchkin, David, "El mandato civil (continuación)", Revista de Derecho Universidad de Concepción, 1940, no 35-36, año IX, pp. 2887-2941.

Stitchkin, David, "El mandato civil (continuación)", Revista de Derecho Universidad de Concepción, 1941, no 37-38, año IX, pp. 2991-3030.

Stitchkin, David, "El mandato civil (continuación)", Revista de Derecho Universidad de Concepción, 1942, n 39-40, año X, pp. 1-58.

STITCHKIn, David, "El mandato civil (continuación)", Revista de Derecho Universidad de Concepción, 1944, $\mathrm{n}^{\circ}$ 47, año XII, pp. 1-38.

Stitchkin, David, "El mandato civil (continuación)", Revista de Derecho Universidad de Concepción, 1944, n 48 , año XII, pp. 137-153.

Stitchkin, David, "El mandato civil", Revista de Derecho Universidad de Concepción, 1944, n 49, año XII, pp. 249-274.

STitchkin, David, "El mandato civil (continuación)", Revista de Derecho Universidad de Concepción, 1945, n 51, año XIII, pp. 1-27.

Stitchkin, David, "El mandato civil (continuación)", Revista de Derecho Universidad de Concepción, 1945, $\mathrm{n}^{\mathrm{O}}$ 52, año XIII, pp. 187-221.

Stitchkin, David, "El mandato civil (continuación)", Revista de Derecho Universidad de Concepción, 1945, $\mathrm{n}^{\circ}$ 53, año XIII, pp. 225-268.

Stitchkin, David, "El mandato civil (continuación)", Revista de Derecho Universidad de Concepción, 1946, n 55, año XIV, pp. 79-121.

STIтchкin, David, "El mandato civil", Revista de Derecho Universidad de Concepción, 1946, n ${ }^{\circ}$, año XIV, pp. 317-347.

Stitchkin, David, Derecho Civil, Editorial Universitaria S.A. Santiago, 1948, Tomo I y II.

StitchKIn, David, "Comentario a sentencia de la Corte Suprema de 18 de 
marzo de 1948", Revista de Derecho y Jurisprudencia, T. XLVI, $2^{\text {a }}$ parte, sección $1^{\circ}$, pp. 94-98. 1950.

Stitchkin, David, El mandato civil, Editorial Jurídica de Chile, Santiago,

StitchKin, David, "Discurso de incorporación de don David Stitchkin Branover como miembro académico de la H. Facultad de Ciencias Jurídicas y Sociales de la Universidad de Concepción", Revista de Derecho Universidad de Concepción, 1961, n 115, año XXIX, pp. 199-205.

Stitchkin, David, "Los Bienes extrapatrimoniales", Revista de Derecho Universidad de Concepción, 1961, $\mathrm{n}^{\circ}$ 115, año XXIX, pp. 3-29.

Stitchkin, David, "El abogado en la Universidad. Conferencia de don David Stitchkin pronunciada el 16 de diciembre de 1966 en el Consejo General del Colegio de Abogados", Revista de Derecho y Jurisprudencia, T. LXIII, $1^{\text {a }}$ parte, pp. 255-265.

Stitchkin, David, "Los contenidos esenciales del derecho de propiedad", en: El campesino, Vol. XCVIII (3): transcrito en Garrido, José; Guerrero, Cristián, VALDÉs María Soledad, Historia de la reforma agraria en Chile, Editorial Universitaria, Santiago, 1978, pp. 211-220.

Stitchkin, David, Prólogo al libro de Bustamante, Luis, El Patrimonio. Dogmática Jurídica, Editorial Jurídica de Chile, Santiago, 1979, pp. 7-11.

Stitchkin, David, Ley, Derecho y Justicia. Cuadernos de Análisis Jurídico no 1, Escuela de Derecho Universidad Diego Portales, Santiago, 1987.

Stitchkin, David, "Adquisición de pagaré", Revista Chilena de Derecho, 1990, Vol. 17, n 1, pp. 211-231.

Stitchkin, David, "La socialización del Derecho", Anuario de Filosofía jurídica y social, 1990, $\mathrm{N}^{\mathrm{o}}$ 8, pp. 401-403.

Stitchrin, David, Prólogo al Libro de: Figueroa Yáñez, Gonzalo, El patrimonio, Editorial Jurídica de Chile, Santiago, 1991.

Stitchkin, David, El Mandato Civil. Editorial Jurídica de Chile, Santiago, 2020, $5^{\text {a }}$ Ed. actualizada por Gonzalo Figueroa.

TAPIA, Orlando, De la responsabilidad civil en generaly de la responsabilidad delictual entre los contratantes. Lexis Nexis, Santiago, 2006, $2^{\mathrm{a}}$ ed.

Universidad de ChILE, "Hoja de vida del funcionario David Stitchkin Branover", documento, s/d., copia obtenida por Oficio UT (O) N² 204/2020 de 16 de junio de 2020, por ley de transparencia.

UnIVERSIDAD DE CONCEPCIÓN, Memoria del Directorio correspondiente al año 1941. 1942, archivo en formato .pdf, disponible en línea: http://www. archivohistoricoconcepcion.cl/assets/memorias_udec/Memoria_UdeC_1941.pdf

Universidad de Concepción (Eds.) Académicos en Sintonía IV, Comentarios en Radio Universidad de Concepción, Ed. Universidad de Concepción, Concepción, 2013.

Venegas, Fernando, Violeta Parra en Concepción y la Frontera del Biobio: 1957-1960. Recopilación, difusión del folklore y desborde creativo. Editorial Universidad de Concepción, Concepción, 2017. 